\title{
Crisis económica y determinantes de la oferta de trabajo femenino en México: 1994-1995
}

\author{
René M. Zenteno*
}

La rápida incorporación de la mujer al mercado de trabajo constituye uno de los temas de investigación de mayor relevancia a la luz de las rápidas transformaciones sociales, económicas y demográficas que ha experimentado México en las uiltimas dos décadas. Entre muchos otros factores, diversos estudios han argumentado sobre la existencia de una estrecha relación entre este proceso de cambio social de la mujeriy la crisis económica que han enfrentado muchas familias mexicanas durante un largo periodo de restructuración productiva. La base de este argumento deriva, en gran medida, de la cuantificación de un fenómeno poco común en México hasta antes de los años ochenta: una creciente participación económica de mujeres en edades adultas avanzadas, con bajo nivel educativo, casadas o en unión libre y, finalmente, de mujeres con hijos pequeños. Es decir, de grupos sociales que tradicionalmente habian manifestado una muy baja propensión a trabajar, fuera del hogar, El presente articulo constituye un estudio de coyuntura cuyo objetivo principal fue evaluar empiricamente la relación entre crisis económica y este nuevo peifil de mano de obra femenina. Para ello se estudiaron los de terminantes de la aferta del empleo urbano femenino durante los primeros meses de la crisis económica más reciente, en contraste con la situación prevaleciente exactamente un año antes. Los datos de la Encuesta Nacional de Empleo Urbano muestran que el ingreso real de los trabajadores, el desempleo abierto y la tasa de ocupación de los hombres en el trabajo asalariado, fueron las condiciones del empleo urbano más afectadas por la recesión. Los análisis multivariados realizados permiten señalar tres resultados importantes. Primero, que los impactos de la crisis sobre la participación económica de hombres y mujeres variaron significativamente entre los centros urbanos más importantes del país. Segundo, que con la excepción de las mujeres con más baja educación, éfectivamente es posible establecer la existencia de una estrecha relación entre la mayor ocupación de mujeres de mayor edad, unidas, y que residen en hogares donde cohabitan niños menores de siete años, con una situación de crisis económica. Tercero, que varios de estos cambios sucedieron no únicamente en mercados laborales donde la participación económica femenina aumentó durante el periodo, sino también en aquéllos en que ésta se mantuvo estable o disminuyó.

\section{Introducción}

En enero de 1994, los mexicanos empezamos a tener más claro que la desigualdad social y económica persistente en el país no era precisamente el argumento más sólido para sostener que estábamos a punto

* Centro de Estudios Estratégicos, Instituto Tecnológico y de Estudios Superiores de Monterrey, campus Guadalajara. 
de tocar las puertas del mundo desarrollado. Ante nosotros se empezaba a revelar la frustrante posibilidad de presenciar el final de uno más de nuestros acostumbrados viajes sexenales a un México que se promete cambiar. Meses después, un asesinato en Tijuana y otro en la Ciudad de México, no sólo confirmaron nuestros temores sino que también nos hicieron recordar nuestra letal capacidad para dirimir diferencias. En diciembre del mismo año, y en menos de una semana, el cataclismo finalmente ocurrió. La mayoría de las familias mexicanas pasaron de ser lectores de una historia trágica a ser protagonistas de la misma. Aunque un poco más tarde de lo que la historia reciente dictaba, el ciclo sexenal había cerrado su acostumbrado círculo.

Contracción de la producción y la demanda, cierre masivo de empresas, desempleo e inflación, volvieron a formar parte de nuestra realidad social y económica. Según datos manejados por Oliveira y García (1997), en 1995 el producto interno del país se redujo 6.9\%; la inflación acumulada llegó a 52\%; el desempleo abierto alcanzó un máximo de $7.6 \%$ durante el mes de agosto, y cerca de 20 mil empresas dejaron de cotizar en el Instituto Mexicano del Seguro Social.

Los datos de la Encuesta Nacional de Empleo Urbano (ENEU) permiten observar los efectos inmediatos de la crisis económica en el agregado de 37 de los centros urbanos más importantes del país (véase el cuadro 1). ${ }^{1}$ La información de este cuadro permite concluir que, al comparar algunos indicadores del mercado de trabajo urbano del trimestre abril.junio de 1995 con los correspondientes al mismo periodo del año anterior, el ingreso real de los trabajadores, el desempleo abierto y la tasa de ocupación de los hombres en el trabajo asalariado, fueron las condiciones del empleo urbano más afectadas por la recesión.

El ingreso real promedio y mediano de la población económicamente activa (PEA) ocupada disminuyó de manera significativa en tan sólo un año. ${ }^{2}$ Este descenso fue igualmente significativo para los hombres y para las mujeres ( 21 y $19 \%$, respectivamente). La gráfica 1 permite apreciar el efecto de este cambio sobre el ingreso per cápita de

${ }^{1}$ La lista de centros urbanos puede apreciarse en el cuadro 2.

${ }^{2}$ Los ingresos fueron deflactados utilizando los índices de precios al consumidor publicados por el Banco de México para la mayoría de las ciudades en que se levanta la información de la ENEU. Para los centros urbanos donde esta información no estaba disponible, se utilizó el índice de precios al consumidor de la ciudad que estuviera más cercana físicamente y clasificada además en la misma zona de salarios mínimos. 
CUADRO 1

Efectos de la crisis económica mexicana sobre algunos indicadores del mercado de trabajo urbano en México (37 centros urbanos), 1994 y 1995

\begin{tabular}{|c|c|c|c|c|}
\hline & \multicolumn{2}{|c|}{ Hombres } & \multicolumn{2}{|c|}{ Mujeres } \\
\hline & 1994 & 1995 & 1994 & 1995 \\
\hline \multicolumn{5}{|c|}{$\begin{array}{l}\text { Ingreso de la PEA ocupada en } \\
\text { la ocupación principal } \\
\text { (pesos de 1994) }\end{array}$} \\
\hline Media & $\$ 1320.1$ & $\$ 1091.9$ & $\$ 913.0$ & $\$ 725.6$ \\
\hline Mediana & $\$ 887.6$ & $\$ 697.8$ & $\$ 665.7$ & $\$ 539.5$ \\
\hline Desviación estándar & $\$ 1982.5$ & \$3 545.1 & $\$ 1154.2$ & $\$ 965.1$ \\
\hline Tasa de desempleo abierto & 3.7 & 6.4 & 3.4 & 6.6 \\
\hline \multicolumn{5}{|l|}{ Tasa de participación } \\
\hline Total & 70.7 & 68.1 & 34.4 & 34.8 \\
\hline En trabajo asalariado & 45.6 & 42.2 & 24.1 & 24.2 \\
\hline En trabajo no asalariado & 25.1 & 25.9 & 10.3 & 10.6 \\
\hline \multicolumn{5}{|c|}{$\begin{array}{l}\text { Número de horas trabajadas } \\
\text { por la PEA ocupada (semana) }\end{array}$} \\
\hline Media & 45.6 & 45.5 & 37.9 & 37.9 \\
\hline Mediana & 46.0 & 46.0 & 40.0 & 40.0 \\
\hline Desviación estándard & 14.7 & 16.0 & 15.3 & 15.7 \\
\hline
\end{tabular}

Fuente: Encuesta Nacional de Empleo Urbano, abril-junio de 1994 y 1995. Datos procesados por el autor.

los hogares, al contrastar la distribución acumulada de la población total de estos centros urbanos en 1995, según la distribución por deciles de los ingresos del hogar observada un año antes. Así, por ejemplo, en el mismo nivel de ingreso por hogar en el que vivía $50 \%$ de la población urbana en 1994, lo hacía $59 \%$ en 1995. El deterioro de los ingresos reales de los hogares empobreció aproximadamente a $10 \%$ de la población urbana nacional, tan sólo en los primeros seis meses de haberse iniciado la crisis económica.

El desempleo abierto en las áreas urbanas del país se expandió de manera considerable, particularmente entre las mujeres cuya tasa casi se dobló. Sin embargo, para nuestra propia sorpresa, los niveles de ocupación y el número de horas trabajadas, no sufrieron grandes alteraciones durante el segundo trimestre de 1995. El único efecto notable fue la contracción de los niveles de ocupación masculina como consecuen- 


\section{GRÁFICA 1}

Distribución acumulada de la población residente en 37 centros urbanos del país, en 1995, según la distribución por deciles del ingreso del hogar (por concepto de trabajo) de 1994 (pesos de 1994)

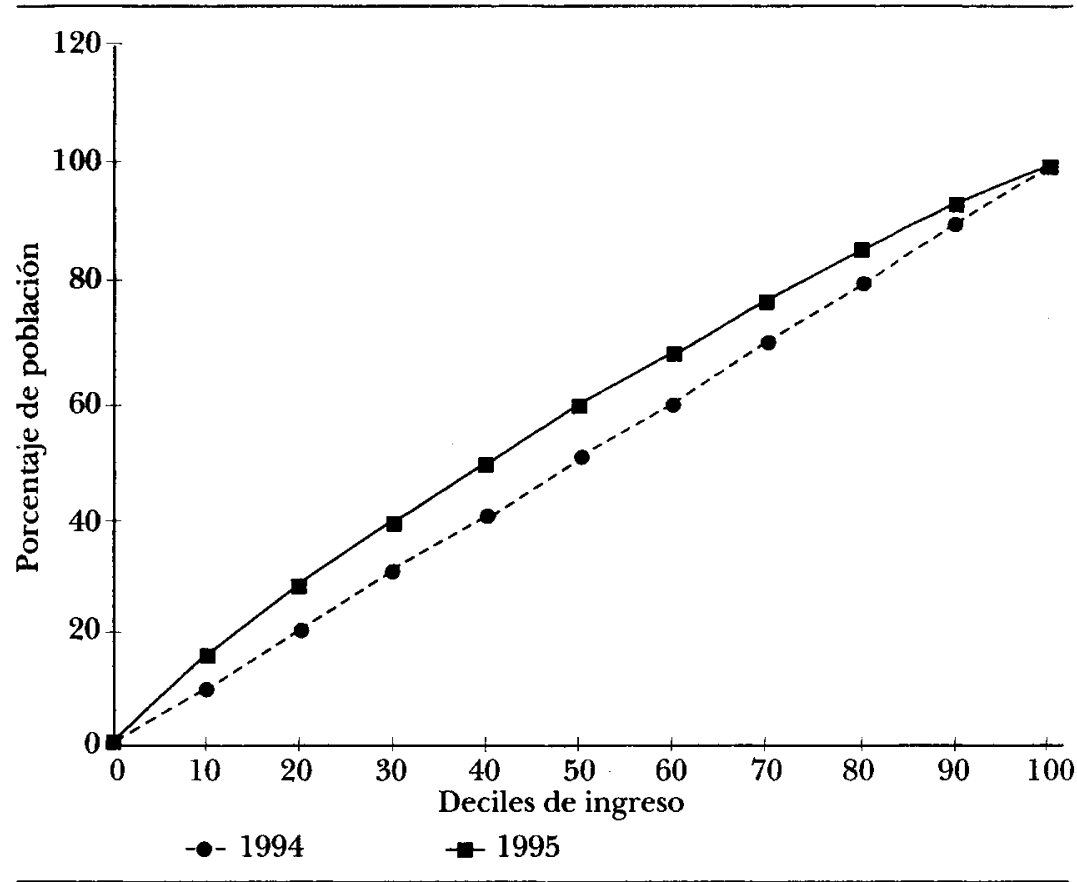

Fuente: Encuesta Nacional de Empleo Urbano, abril-junio de 1994 y 1995. Datos procesados por el autor.

cia de una caída absoluta de $4 \%$ en el trabajo asalariado. ${ }^{3}$ Este resultado coincide con los hallazgos de Pacheco y Parker (1997) quienes encontraron, analizando prospectivamente los paneles muestrales de la Encuesta Nacional de Empleo Urbano, que el porcentaje de desempleo abierto como consecuencia de despidos no aumentó de manera significativa en 1995 en comparación con lo observado en 1986.

¿Fue el deterioro de los ingresos reales resultado de una reducción en el número de horas trabajadas? Una comparación general muestra que la respuesta a esta pregunta es negativa. El número promedio de ho-

${ }^{3}$ La tasa de participación de los hombres en trabajo no asalariado se mantuvo prácticamente igual. 
ras trabajadas entre la PEA ocupada masculina se mantuvo entre 45 y 46 horas a la semana, aunque esto se logró con un incremento en la varianza. El número promedio de horas trabajadas tampoco muestra que las mujeres se vieron obligadas a trabajar menos o más horas de lo que lo hacían en un año atrás. La información de la ENEU permite postular que la caída de los ingresos reales de los hogares estuvo débilmente relacionada, en el agregado nacional urbano, con menores niveles de ocupación de la población y con una reducción en el número de horas trabajadas.

La información de la ENEU revela una mayor incorporación de las mujeres a la fuerza de trabajo durante los primeros meses de 1995, no tanto como consecuencia de cambios importantes en sus niveles de ocupación sino como resultado de un aumento significativo en sus tasas de desempleo abierto. El objetivo del presente trabajo es precisamente analizar cuáles fueron los efectos más importantes de la crisis económica reciente sobre los determinantes de la oferta de trabajo femenino en las ciudades más importantes del país. El trabajo empírico está orientado a verificar, en un análisis de corto plazo, la argumentación que sostiene que la creciente incorporación al mercado de trabajo de mujeres cuya participación económica había sido tradicionalmente baja (las menos jóvenes, las de menor instrucción formal, las casadas o unidas, y las mujeres con niños pequeños), ha sido resultado de una estrategia de los hogares para hacer frente a las dificultades económicas.

La evaluación de corto plazo se basa en la información de la ENEU de los segundos trimestres naturales de 1994 y 1995. Después de presentar la revisión de la literatura pertinente, los determinantes de la oferta de mano de obra femenina se analizan en dos niveles. Por un lado, se examina el agregado de 37 centros urbanos del país para los cuales se cuenta con información comparable para este periodo. Por el otro, se realiza un análisis comparativo de las áreas urbanas de la Ciudad de México, Monterrey y Tijuana. Estos centros urbanos fueron seleccionados no sólo por su importancia económica'en el contexto nacional y porque representan contextos de desarrollo muy particular, sino también debido a los distintos efectos que la crisis económica tuvo sobre la participación económica en cada una de ellas.

\section{Crisis y participación femenina}

Durante los últimos 15 años, México ha experimentado un largo periodo de ajuste estructural y recurrentes crisis económicas. La crisis 
de la deuda a principios de los años ochenta, significó para el país el abandono de una estrategia de desarrollo basada en un modelo industrial proteccionista de sustitución de importaciones, por un modelo de apertura comercial y de fomento a la industria de exportación. Las nuevas reformas económicas impulsadas por el gobierno mexicano han tenido dos consecuencias importantes en términos de empleo: un incremento del empleo en empresas manufactureras exportadoras y una expansión de las actividades económicas informales. Estas respuestas no han sido neutrales en términos de género. La apertura de la economía mexicana al comercio y a la inversión internacional ha significado una mayor participación de la mujer en actividades manufactureras (Oliveira y Roberts, 1993). Igualmente, las mujeres se han incorporado en mayor medida en actividades económicas informales como consecuencia de la restructuración económica y del deterioro en los ingresos reales de los hogares (Escobar y Roberts, 1991; Oliveira y Roberts, 1993).

Como es de esperarse, lo anterior se ha reflejado en un incremento de la participación económica femenina en trabajo extradoméstico. La mejor estimación disponible poco antes de la crisis económica de principios de los años ochenta, en 1979, ubica la tasa de participación femenina nacional en $21.5 \%$. Doce años más tarde esta tasa había aumentado a 31.5\%, y en 1995 su valor se estimó en $34.5 \%$ (García y Oliveira, 1996).

No obstante este patrón general de incremento de la participación femenina en el trabajo extradoméstico, existe una gran heterogeneidad regional y local en lo que respecta a los niveles de incorporación de las mujeres, y en la magnitud de sus aumentos. A un nivel regional esta heterogeneidad ha sido asociada con la relocalización territorial y sectorial de la fuerza de trabajo en el país (García, 1989; Negrete, 1989); y a un nivel local con el tipo de especialización económica de las ciudades (Fussell y Zenteno, 1996), y con los mayores o menores grados de diversificación económica sectorial que han alcanzado las estructuras productivas urbanas del país (Oliveira, 1989). Como veremos más adelante, la información agregada de los 37 centros urbanos incluidos en la ENEU no permite apreciar la variedad de situaciones en que la participación económica se vio afectada por la crisis económica.

Diversos estudios sobre hogares y trabajo femenino han revelado la existencia de conflictos importantes entre los valores normativos y el cambio socioeconómico asociado con la creciente salida de 
la mujer al mercado de trabajo. No obstante, cambios en la estructura social y familiar, así como la persistente restructuración y crisis económica, han contribuido a un incremento significativo de la oferta de trabajo femenino. Por un lado, García y Oliveira (1994) encontraron que mujeres con altos niveles educativos han entrado al mercado de trabajo independientemente de las condiciones de la economía, sin embargo, la mayoría de las mujeres mexicanas no muestran todavía una marcada preferencia por participar en la actividad económica extradoméstica. Esta tensión entre el trabajo doméstico y el extradoméstico de la mujer es ejemplificada por la tendencia de las mujeres de retirarse de la fuerza de trabajo cuando sus hijos son pequeños (González de la Rocha, 1994; Benería y Roldán, 1987). Las mujeres muestran también una elevada intermitencia en los patrones de entrada y salida al mercado de trabajo (Cerrutti, 1997). Por el otro, cambios recientes en los patrones de reproducción de la familia, tales como aumentos en las tasas de separación y divorcio, en la migración masculina y en las jefaturas de hogar femeninas, han repercutido en una mayor incorporación de las mujeres a la oferta de trabajo.

La mayor participación de las mujeres en la fuerza de trabajo también ha estado estrechamente relacionada con la necesidad de generar ingresos adicionales en muchos hogares afectados por la restructuración económica (Lustig, 1990; García y Oliveira, 1994). La hipótesis que vincula crisis y restructuración económica con una mayor incorporación de la mujer al mercado de trabajo, ha sido derivada en varios estudios como resultado de observar una creciente participación económica de mujeres que tradicionalmente habían mostrado muy bajas probabilidades de hacerlo: mujeres en unión y/o con hijos (García y Oliveira, 1992 y 1994; Rubin-Kurtzman, 1993). Además, estas mujeres en edades más avanzadas se reincorporan o interrumpen sólo por periodos breves sus trayectorias laborales (Cruz, 1994). Este patrón de cambio en el perfil de la mano de obra femenina ha tenido lugar en contextos regionales y locales muy diversos en México, y aun en periodos de relativo crecimiento económico.

El objetivo de este trabajo es precisamente verificar esta hipótesis en un periodo de severa crisis económica, como fueron los primeros meses de 1995. De ser cierta esta hipótesis, los efectos recesivos de la economía sobre el nivel de vida de las familias debieron haber repercutido en una mayor participación de mujeres adultas, con bajos nive- 
les educativos, casadas o unidas, y de mujeres viviendo en hogares donde cohabitan niños pequeños. ${ }^{4}$

Crisis, participación económica y mercados locales de trabajo: cuantificando la heterogeneidad

En la introducción de este trabajo se pudo constatar que la crisis económica repercutió notablemente en un aumento del desempleo abierto entre la población de ambos sexos. Sin embargo, con la excepción del trabajo asalariado entre los hombres, las tasas de ocupación de la población económicamente activa (PEA) no mostraron variaciones importantes entre 1994 y 1995. ¿Qué tan generalizables son estos resultados para cada uno de los centros urbanos incluidos en esta encuesta?

Con el fin de contestar esta pregunta de manera adecuada, se calcularon diversos modelos de regresión logística para estimar los logaritmos de los momios (log-odds) de estar desempleado ${ }^{5}$ y de estar trabajando. Además, con el fin de conocer mejor la condición de empleo, se intentó refinar el análisis diferenciando entre la inserción en trabajo asalariado y en trabajo no asalariado. Esta diferenciación conceptual y empírica de la participación económica en México es de gran relevancia no sólo porque ambos tipos de inserción laboral enmarcan condiciones laborales muy desiguales, especialmente en tre las mujeres, sino también porque existe una marcada heterogeneidad en su importancia relativa entre los mercados de trabajo urbano del país (Christenson, García y Oliveira, 1989; García y Oliveira, 1994; Fussell y Zenteno, 1996). Estas cuatro posibilidades se contrastaron con la misma categoría de referencia: la población económicamente

\footnotetext{
${ }^{4}$ Evidentemente es imposible suponer que todas las mujeres de 12 años y más que habitan en hogares con niños (as) pequeños(as) tienen efectivamente una responsabilidad en el cuidado de estos menores. Como la ENEU registra la relación de parentesco con el jefe (a) del hogar, sólo cuando la mujer es jefa del hogar es posible conocer directamente si los menores de edad son sus hijos o no. Evidentemente, es posible suponer sin grandes dificultades que las cónyuges de los jefes son las madres de los hijos (as) de éste. Sin embargo, es imposible conocer quién es la madre de todos los pequeños cuya relación de parentesco con el jefe es otra que la de hijo o hija. Por lo tanto, se consideró necesario usar la variable "presencia de menores de siete años en el hogar" como proxy a una situación general de cuidado de hijos(as) en el hogar, en lugar de excluir a todas las mujeres que no fueran ya sea jefas de hogar o cónyuges.

${ }^{5}$ Se refiere a la definición estándard de desempleo abierto.
} 
inactiva. Los modelos logísticos hicieron depender la participación de los siguientes factores: el mercado de trabajo local, el año de la encuesta y la interacción de ambas.

El cuadro 2 presenta las razones de momios (odds ratios), calculadas con los coeficientes beta de estas regresiones logísticas. ${ }^{6}$ Las interacciones entre mercados locales de trabajo y año fueron estadísticamente significativas en todos los casos, indicando con ello que los efectos inmediatos de la crisis económica sobre los niveles de desempleo abierto y de ocupación no ocurrieron en la misma dirección en todos los contextos urbanos del país. Que las variaciones en el comportamiento de la economía nacional no hayan tenido un efecto homogéneo (por lo menos de manera inmediata) sobre los niveles de participación de los mercados de trabajo urbano local, indica claramente que existen vinculaciones muy diversas y complejas entre las economías locales y la economía nacional. Este hallazgo pone en evidencia la importancia de no asumir que los resultados encontrados en el análisis del conglomerado nacional pueden fácilmente extenderse a todas las ciudades del país, y constituye también una nota de precaución para los estudios que a partir de la investigación de una o dos economías locales, intentan generalizar sus resultados para todo el país. ${ }^{7}$

El comportamiento más homogéneo entre las ciudades se puede observar en el desempleo abierto, pues la tendencia fue -en forma casi general- hacia su incremento. Los únicos centros urbanos donde el desempleo abierto disminuyó fueron Matamoros (ambos sexos) y Monclova y Oaxaca (sólo mujeres). A pesar de este patrón de aumento en el desempleo abierto de casi todas las ciudades, las magnitudes de los cambios variaron de forma significativa entre las mismas. Mientras que la crisis económica repercutió en un aumento de sólo $10 \%$ en la propensión de estar desempleado entre los hombres residentes en Acapulco, este incremento fue de $600 \%$ en Morelia. Las mujeres mostraron un contraste más dramático pues, en tan sólo un año, aquellas residentes en Cuernavaca aumentaron 16 veces su propensión a estar desempleadas.

\footnotetext{
${ }^{6}$ La metodología para el cálculo de las razones de momios puede verse en Zenteno (1995b: capítulo 4).

${ }^{7}$ Una de las ventajas de la Encuesta Nacional de Empleo Urbano es que esta importante fuente de información permite apreciar las variaciones existentes entre distintos contextos económicos y laborales urbanos del país. Uno de los retos a corto plazo es, sin embargo, poder conceptualizar y medir la naturaleza de la heterogeneidad urbana del país.
} 


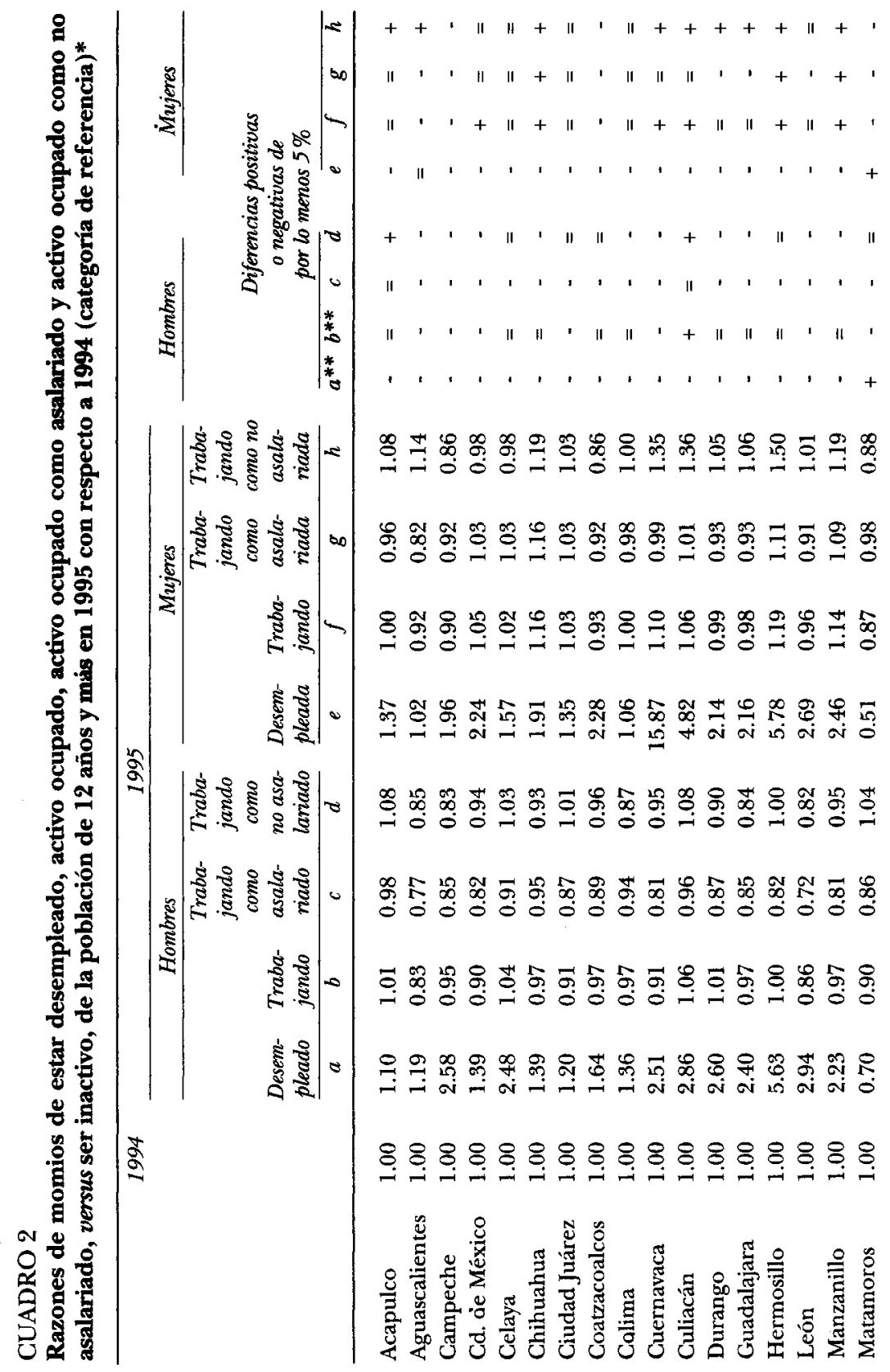




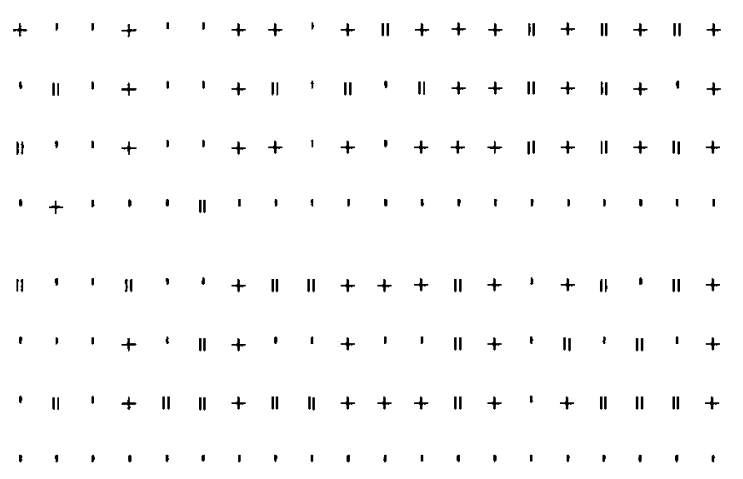

요용

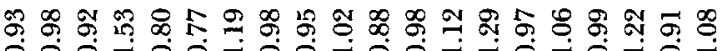

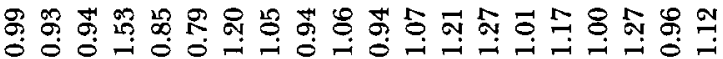

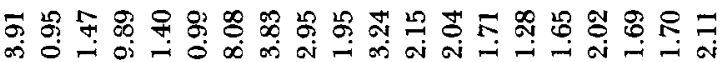

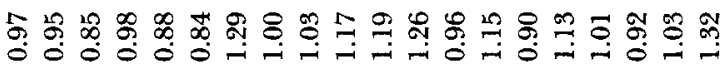

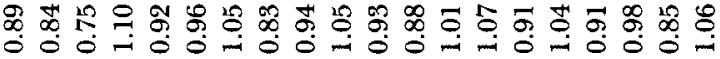

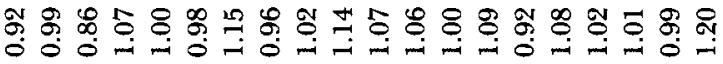

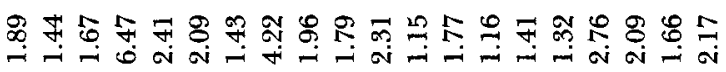
\&\& \& \& \& \& \& \& \& \& \& \& \& \& \& \& \& \& \&

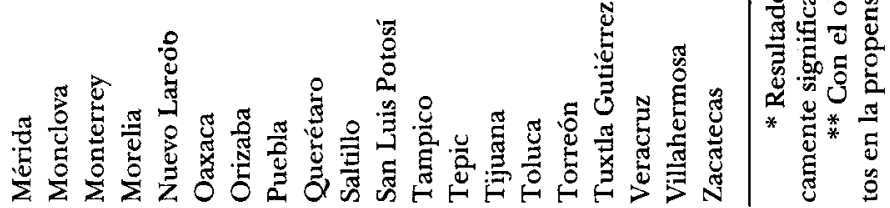


Dado que en México no existe seguro de desempleo, y de que la mayoría de las familias en el país no puede cubrir los costos que significa que alguno(s) de sus miembros estén buscando trabajo por un periodo largo de tiempo, la población se ve obligada a emplearse de cualquier forma con el fin de generar algún tipo de ingreso. Con ello, las ocupaciones no asalariadas representan, en la mayoría de las ocasiones, un desempleo encubierto o subempleo. Este tipo de empleo se caracteriza por su naturaleza irregular en términos de ingreso, de estabilidad y de prestaciones laborales. En 20 de los 37 centros urbanos del país, la crisis económica reciente repercutió en un aumento del empleo femenino en actividades económicas no asalariadas, mientras que sólo en nueve de ellos los hombres experimentaron el mismo cambio. La situación crítica de los hombres queda en evidencia al observar que los niveles de ocupación de éstos en el trabajo no asalariado disminuyó en 16 ciudades.

Los resultados restantes muestran con bastante claridad las grandes diferencias por género en lo que respecta a las consecuencias de la crisis económica. Aunque los cambios en los niveles de ocupación general, y en el trabajo asalariado y no asalariado, variaron de manera significativa entre las ciudades, es posible apuntar que los efectos de la crisis fueron mucho más penetrantes en la PEA ocupada masculina que en la femenina. En los casos de muchas ciudades del país, la disminución significativa del empleo asalariado masculino parece haber sido compensada por una expansión del trabajo no asalariado de las mujeres.

Los efectos diferenciales por género de la crisis económica podrían resumirse de la siguiente manera. Aunque el número de contextos urbanos en donde la ocupación disminuyó significativamente es el mismo para la población de ambos sexos (10 de las 37 ciudades), la población femenina incrementó su nivel de empleo en 16 ciudades. Los hombres pudieron hacer lo mismo sólo en nueve centros urbanos. La ocupación en el trabajo asalariado de los hombres muestra una situación más dramática, al haber disminuido ésta en poco más de dos terceras partes de las ciudades. Esta tendencia puede observarse en menos de la mitad de las ciudades en el caso de las mujeres. Es importante también notar que el número de ciudades en donde hubo un incremento del empleo asalariado de las mujeres, dobla la cifra correspondiente a la de los hombres.

Como se mencionó anteriormente, los efectos de la crisis económica sobre la participación económica no pueden generalizarse a todos 
los centros urbanos del país, a pesar de encontrar patrones semejantes en muchos de éstos. ¿Cuáles fueron entonces los mercados de trabajo más afectados por la crisis económica de diciembre de 1994? Indudablemente podemos situar en el extremo más crítico a aquellas ciudades donde aumentó el desempleo y disminuyeron los niveles de ocupación para ambos sexos: Campeche, Monterrey y Nuevo Laredo. Estas tres ciudades representan economías muy disímiles como para intentar explicar su comportamiento de manera conjunta. Las contracciones de la demanda interna siempre han tenido un efecto negativo inmediato sobre la economía y el empleo de Monterrey, pues su producción industrial continúa teniendo una fuerte orientación al mercado nacional. La economía de Nuevo Laredo, el centro fronterizo más importante del país en lo que al movimiento de mercancías entre México y Estados Unidos se refiere, muy probablemente se vio afectada por la disminución drástica de las importaciones. La situación de Campeche es dificil de explicar para un autor con escaso conocimiento sobre su economía.

En el otro extremo de la situación anterior se encuentran Orizaba, Tijuana y Zacatecas. Estas tres ciudades, también con bases económicas disímiles, fueron afectadas por la crisis económica sólo en el rubro del desempleo, pues la ocupación de hombres y mujeres aumentó tanto en el trabajo asalariado como en el no asalariado. Durante la pasada década, Tijuana mostró que si bien las continuas devaluaciones de la moneda mexicana afectaron la capacidad de consumo de sus pobladores en Estados Unidos, éstas significaron un gran impulso a su economía al volverse más competitivos sus precios y mano de obra para los consumidores e inversionistas extranjeros, respectivamente (Zenteno, 1995a). Orizaba y Zacatecas constituyen una enigma para el autor, más allá de poder argumentar que los resultados observados en los niveles de empleo muestran en apariencia una débil integración de sus economías con la economía nacional.

Analizar la situación de las restantes ciudades sería una tarea muy extensa, y rebasa, además, los objetivos mismos de este artículo. Centremos nuestra atención sobre las tres ciudades seleccionadas para nuestro análisis debido a su importancia económica: la Ciudad de México, Monterrey y Tijuana. Estos tres centros urbanos del país experimentaron cambios muy distintos en la participación económica de su población durante el periodo analizado.

La Zona Metropolitana de la Ciudad de México (ZMCM) representa la economía urbana más grande y diversificada de México. La 
capital del país no sólo genera la mayoría de la producción manufacturera nacional orientada al mercado interno, cuenta con un sector moderno de servicios al productor, y alberga la infraestructura gubernamental y social de mayor tamaño absoluto y relativo en el territorio nacional. Durante los primeros meses de 1995, la participación económica de los hombres y de las mujeres había sido afectada de manera muy distinta. Por un lado, la propensión a trabajar de los hombres había disminuido en $10 \%$, principalmente como consecuencia de una reducción de $18 \%$ en el trabajo asalariado. A pesar de esto, el incremento del desempleo abierto masculino fue uno de los más bajos entre las ciudades consideradas (39\%). Por otro lado, la tendencia entre las mujeres residentes en la ZMCM muestra una mayor incorporación a la fuerza de trabajo, pues si bien sus niveles de participación en trabajo asalariado y no asalariado no sufrieron modificación significativa alguna, el desempleo abierto femenino creció en más de 200 por ciento.

Monterrey es el segundo centro industrial del país. Su peculiar historia ha derivado en una economía y sociedad con gran penetración del modo de producción capitalista. Por lo mismo, las crisis económicas del país dejan sentir efectos más agudos en esta ciudad norteña, tal y como ocurrió durante la pasada década (Pozos, 1995). Como se vio anteriormente, esta vez no fue la excepción. Monterrey fue una de las tres ciudades más afectadas por la crisis económica que dio inicio a finales de 1994. El desempleo abierto de hombres y mujeres se expandió en 67 y $47 \%$ respectivamente. Esta ciudad presentó la segunda mayor disminución tanto en el total del empleo masculino (después de Aguascalientes) como en el trabajo asalariado entre los hombres (después de León). La ocupación femenina mostró una tendencia igualmente descendente tanto en el empleo asalariado como en el no asalariado. Podría argumentarse que este centro urbano nacional es quizás el ejemplo más conspicuo de la alta correspondencia entre la demanda de bienes y servicios generada por el trabajo asalariado, y la oferta de actividades económicas no asalariadas (la mayoría de ellas informales).

Tijuana es la ciudad más importante del país en lo que respecta al nuevo modelo de desarrollo industrial exportador. Durante los últimos 15 años, esta ciudad fronteriza ha experimentado un crecimiento económico sostenido, en gran parte como consecuencia de una expansión de la industria maquiladora de exportación y de los servicios productivos y sociales modernos. Con ello, las condiciones laborales han mejorado sustancialmente en este centro urbano fronterizo (Hualde y Zenteno, 1996). Aunque la tasa de desempleo abierto 
aumentó significativamente en Tijuana entre 1994 y 1995, particularmente entre las mujeres, la información de la ENEU muestra un panorma de la ocupación completamente opuesto al de Monterrey. Las posibilidades de estar empleado en esta ciudad fronteriza se incrementaron, entre los hombres y las mujeres, en 9 y $27 \%$ respectivamente. Este cambio fue igualmente significativo en el trabajo asalariado como no asalariado para la población de ambos sexos, aunque el crecimiento en términos relativos de este último tipo de empleo fue superior al primero. Es posible entonces observar que la población de Tijuana experimentó las repercusiones menos graves de la crisis económica más reciente en lo que a la participación económica se refiere. ${ }^{8}$

En resumen, la ZMCM, Monterrey y Tijuana no sólo representan economías muy diversas, sino también escenarios distintos de los efectos de la crisis económica sobre el empleo masculino y femenino. La capital del país representa un contexto donde el deterioro de la participación masculina estuvo acompañado por una estabilidad en los niveles de ocupación de las mujeres pero con incremento en su desempleo abierto, es decir, por una mayor incorporación de la mujer a la fuerza de trabajo. La situación de los hombres de Monterrey no fue muy distinta a la de sus similares de la ZMCM. Sin embargo las mujeres regiomontanas, a diferencia de las de la Ciudad de México, no pudieron sostener los niveles de ocupación que tenían un año antes, esto tanto en el trabajo asalariado como en el no asalariado. Tijuana presenta una situación completamente opuesta a la de la ZMCM y Monterrey, pues aunque el desempleo abierto aumentó entre 1994 y 1995, los niveles de ocupación también se incrementaron de manera significativa, particularmente entre las mujeres.

Los determinantes de la oferta de empleo femenino

\section{El agregado urbano}

Al analizar los determinantes de la oferta de empleo femenino durante los primeros meses de la reciente crisis económica, el presente trabajo intenta probar la hipótesis que vincula un contexto de crisis eco-

${ }^{8}$ Morelia, la única ciudad que superó a Tijuana en lo que respecta a crecimiento del trabajo asalariado, muestra sin embargo los incrementos más altos en desemṕleo abierto entre las ciudades aquí analizadas. 
nómica con la salida al mercado de trabajo de mujeres con muy baja propensión a trabajar. Particularmente mujeres con bajos niveles educativos, en edades adultas avanzadas, casadas o unidas, y residiendo en hogares que cuentan con niños pequeños. Antes de analizar de manera específica cada una de las ciudades seleccionadas, y con el fin de contar con un marco de referencia general, se decidió primero analizar los determinantes de la oferta de empleo femenino en el agregado urbano de las 37 localidades urbanas incluidas en la ENEU en 1994 y 1995.

El cuadro 3 presenta el modelo de oferta de mano de obra femenina con el que se propone contrastar empíricamente la relación entre crisis y participación femenina. En él se incluye la edad, el último año de educación formal, la condición deijefatura de hogar, el estado marital al momento de la encuesta, la presencia de niños(as) menores de 7 años en el hogar, y el tipo de hogar en que residen las mujeres. Lajustificación de este modelo sociodemográfico de oferta de mano de obra, así como una comprobación empírica del mismo se presenta en otro trabajo del autor (Fussell y Zenteno, 1996). Con el único propósito de ilustrar la robustez del modelo, las primeras ocho columnas del cuadro 3 muestran las propensiones relacionadas con las distintas formas de participación en la fuerza de trabajo para cada uno de los años analizados. Con excepción del caso del desempleo abierto que nunca ha sido analizado a la luz de este modelo conceptual (al menos para el caso mexicano), esta información no muestra ninguna novedad respecto a nuestro conocimiento sobre quiénes son las mujeres con más probabilidades de trabajar: jóvenes, educadas, ijefas de hogar, solteras, y aquellas que viven en hogares extendidos y en donde no residen menores de siete años de edad.

Es interesante observar la relevancia empírica del modelo sociodemográfico de oferta de mano de obra femenina en el caso del desempleo abierto. Con la excepción de la variable presencia de menores de edad en 1994, el resto de factores están relacionados de manera significativa con la propensión de estar desempleado. Así, son las mujeres de 25 a 34 años de edad, las de mayor nivel educativo, jefas de hogar, solteras, que viven en hogares extendidos, y residen en hogares donde hay presencia de menores, las más probables de estar buscando empleo de forma activa.

La información pertinente al desarrollo de los objetivos de este trabajo puede observarse en la segunda parte del cuadro 3. En ella se 


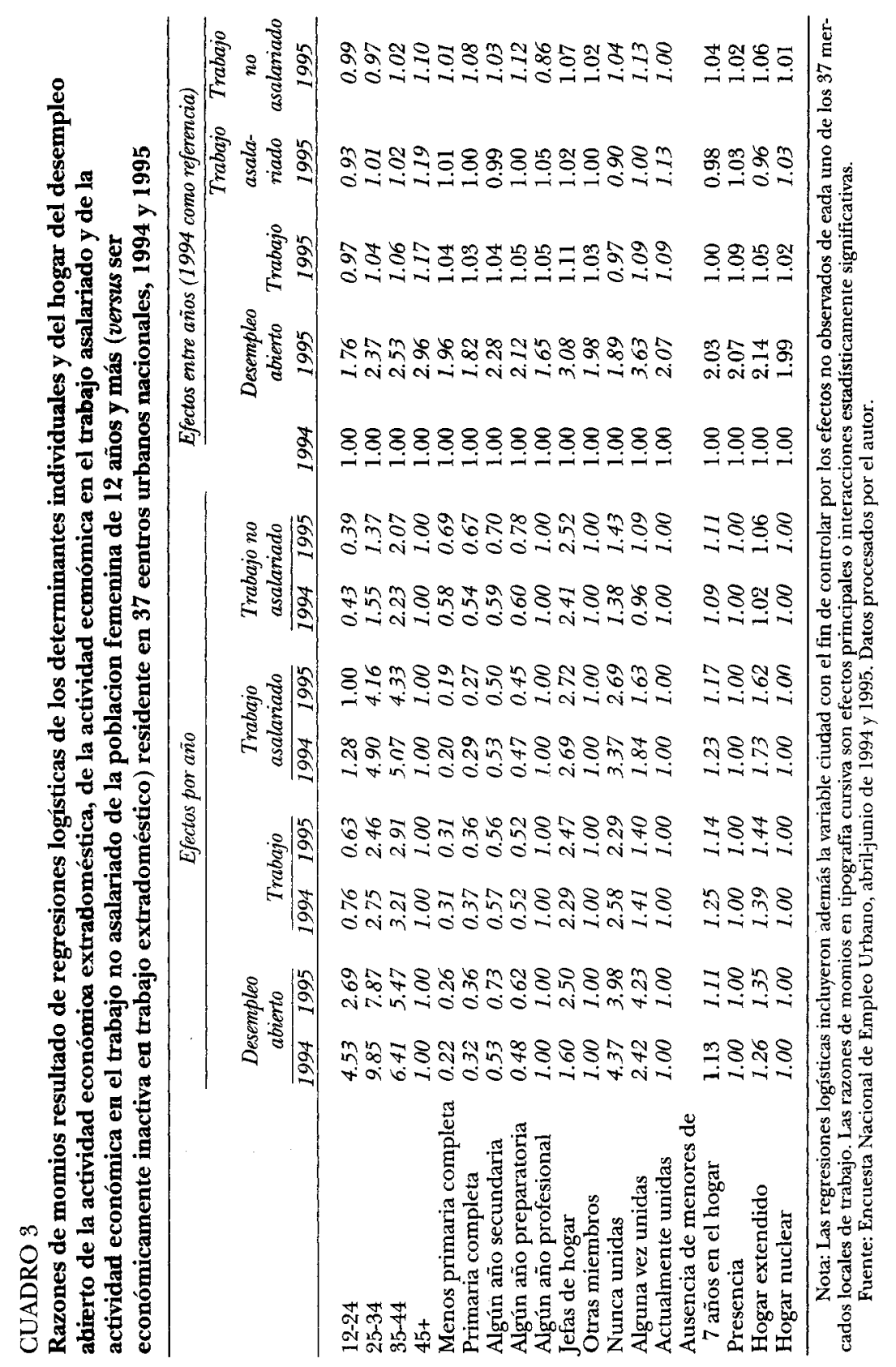


muestra información que indica los cambios ocurridos en la oferta de mano de obra femenina entre 1994 y 1995, así como la importancia estadística de los cambios. Por razones de espacio la discusión de los resultados se limita a establecer la validez empírica de las hipótesis descritas anteriormente.

La edad fue el único determinante de la oferta de trabajo femenino que experimentó cambios significativos en cada uno de los tipos de participación económica. Los resultados son bastante claros sobre el aumento del desempleo de las mujeres en todos los grupos de edad, como consecuencia de la recesión. Sin embargo, es importante hacer notar que el crecimiento más significativo en los niveles de desempleo ocurrió entre las mujeres de $\mathbf{4 5}$ años y más. La mayor incorporación a la fuerza de trabajo de las mujeres en edades adultas avanzadas queda claramente en evidencia no sólo por la expansión de su desempleo, sino también porque su nivel de ocupación aumentó más que el de cualquier otro grupo de mujeres: $17 \%$ entre 1994 y 1995. Aún más interesante es observar que la mayor ocupación de estas mujeres ocurrió tanto en empleo asalariado como no asalariado (19 y $10 \%$, respectivamente). El incremento del trabajo asalariado entre las mujeres de 45 años y más es sorprendente, especialmente si se toma en consideración que el efecto de la educación está controlado en el modelo estadístico. Una posible explicación es que exista una mayor disposición de estas mujeres a trabajar por un menor salario, y que por lo tanto se esté observando realmente una transformación en la demanda de trabajo.

Los resultados de la variable educación muestran que los cambios fueron significativos en el caso del desempleo abierto y del trabajo no asalariado. Los mayores aumentos en el desempleo abierto ocurrieron entre las mujeres con algún año de educación secundaria o con algún año de educación preparatoria. A pesar de que el análisis transversal muestra que las mujeres con mayor instrucción formal son las que tienen más probabilidades de estar desempleadas, los resultados muestran que la crisis económica repercutió menos en el desempleo de las mujeres con educación profesional. Esto indica claramente que la educación universitaria es un importante seguro contra el desempleo en situaciones económicas apremiantes.

$\mathrm{El}$ análisis de regresión arraja evidencias sobre un crecimiento positivo pero insignificante del empleo femenino en todos los niveles educativos. Contrario a lo planteado en nuestras hipótesis, la información del conjunto nacional urbano no indica que las mujeres con 
más bajo nivel de instrucción formal aumentaron significativamente sus niveles de empleo durante los primeros meses de la crisis económica. En el caso de la educación la única interacción estadísticamente significativa tuvo lugar en el ámbito del trabajo no asalariado, lo cual muestra la importancia analítica de diferenciar el tipo de inserción laboral. Así, las mujeres con algún año de preparatoria incrementaron su participación en el trabajo no asalariado en $12 \%$ entre 1994 y 1995, mientras que aquéllas con primaria completa les siguieron con $8 \%$ de cambio positivo. El trabajo no asalariado de mujeres con una formación superior a la preparatoria fue el único que disminuyó de manera importante durante el periodo analizado.

Como se vio anteriormente, lasijefas de hogar tienen una mayor propensión a participar económicamente (como ocupadas y desocupadas) que otras mujeres del hogar. El análisis temporal indica además que, entre 1994 y 1995, las jefas de hogar aumentaron en mayor medida su participación económica que aquellas que no lo son. La interacción no fue estadísticamente significativa debido a que los momios de participación de ambos grupos parte de valores muy altos en 1995. La apremiante situación económica de los hogares dirigidos por mujeres queda en evidencia no sólo por los aumentos en sus niveles de ocupación, sino también por el elevado crecimiento de su desempleo abierto: 300 por ciento.

Los resultados según el estado marital de las mujeres muestran una situación muy interesante. Por un lado, aunque el desempleo abierto se incrementó entre todas las mujeres, el cambio más elevado ocurrió entre aquellas alguna vez unidas (separadas, divorciadas y viudas). Por el otro, los resultados confirman no sólo un mayor aumento del empleo de las mujeres unidas en comparación con las solteras, sino también un mayor incremento de las mujeres alguna vez unidas. Ambos grupos de mujeres elevaron su propensión a estar empleadas en 9\%. Lo interesante deriva de la diferenciación según el tipo de inserción. Mientras que las mujeres unidas aumentaron su participación en el trabajo asalariado en $13 \%$, las mujeres alguna vez unidas lo hicieron en el mismo porcentaje pero en el trabajo no asalariado.

A pesar de que la interacción no fue significativa, los resultados de la regresión logística muestran que aunque el desempleo abierto aumentó tanto entre las mujeres que residen en hogares donde no viven menores de siete años de edad, como entre aquellas que lo hacen en hogares donde sí los hay, la participación económica creció más entre 
las últimas que entre las primeras. Este resultado muestra con bastante claridad que en situaciones económicas adversas, la presencia de menores en el hogarijuega un papel inhibitorio menos importante sobre la inserción de la mujer en el mercado de trabajo.

Si bien la diferencia es mínima, el desempleo abierto se elevó más entre los hogares extendidos que entre los nucleares. Lo mismo puede afirmarse de la ocupación general, sin embargo, es interesante observar que el mayor empleo de las mujeres que viven en hogares extendidos ocurrió por completo en el trabajo no asalariado, pues su participación en el trabajo asalariado incluso disminuyó durante el periodo analizado. La interacción significativa observada en el trabajo asalariado indica la existencia de un mayor crecimiento del empleo femenino en los hogares nucleares que en los extendidos.

\section{Zona Metropolitana de la Ciudad de México, Monterreyy Tijuana}

Como se mencionó anteriormente, los efectos de la crisis económica fueron muy variados en la ZMCM, Monterrey y Tijuana. En la ZMCM fue posible observar una estabilidad en los niveles de ocupación de las mujeres pero también un incremento en su desempleo abierto. En Monterrey el aumento en el desempleo abierto de las mujeres fue acompañado por una reducción en sus niveles de empleo. Finalmente, la mayor incorporación de la mujer tijuanense a la fuerza de trabajo fue resultado tanto de incrementos en su desempleo abierto como en sus tasas de ocupación.

El cuadro 4 permite apreciar las variaciones temporales de los determinantes de la oferta de trabajo femenino en estas distintas situaciones de cambio en la participación femenina. La comparación es una vez más con respecto a los niveles de desempleo y empleo prevalecientes durante el segundo trimestre de 1994, y la lectura de los resultados se centra únicamente en responder a los objetivos planteados en este trabajo.

Las razones de momios por ciudad y tipo de inserción laboral confirman sustancialmente los resultados encontrados en el agregado nacional urbano, aunque existen algunas excepciones importantes en Monterrey. Los resultados del análisis multivariado permiten sostener que las mayores propensiones a trabajar de mujeres adultas, unidas, y de aquéllas que residían en hogares con menores de siete 


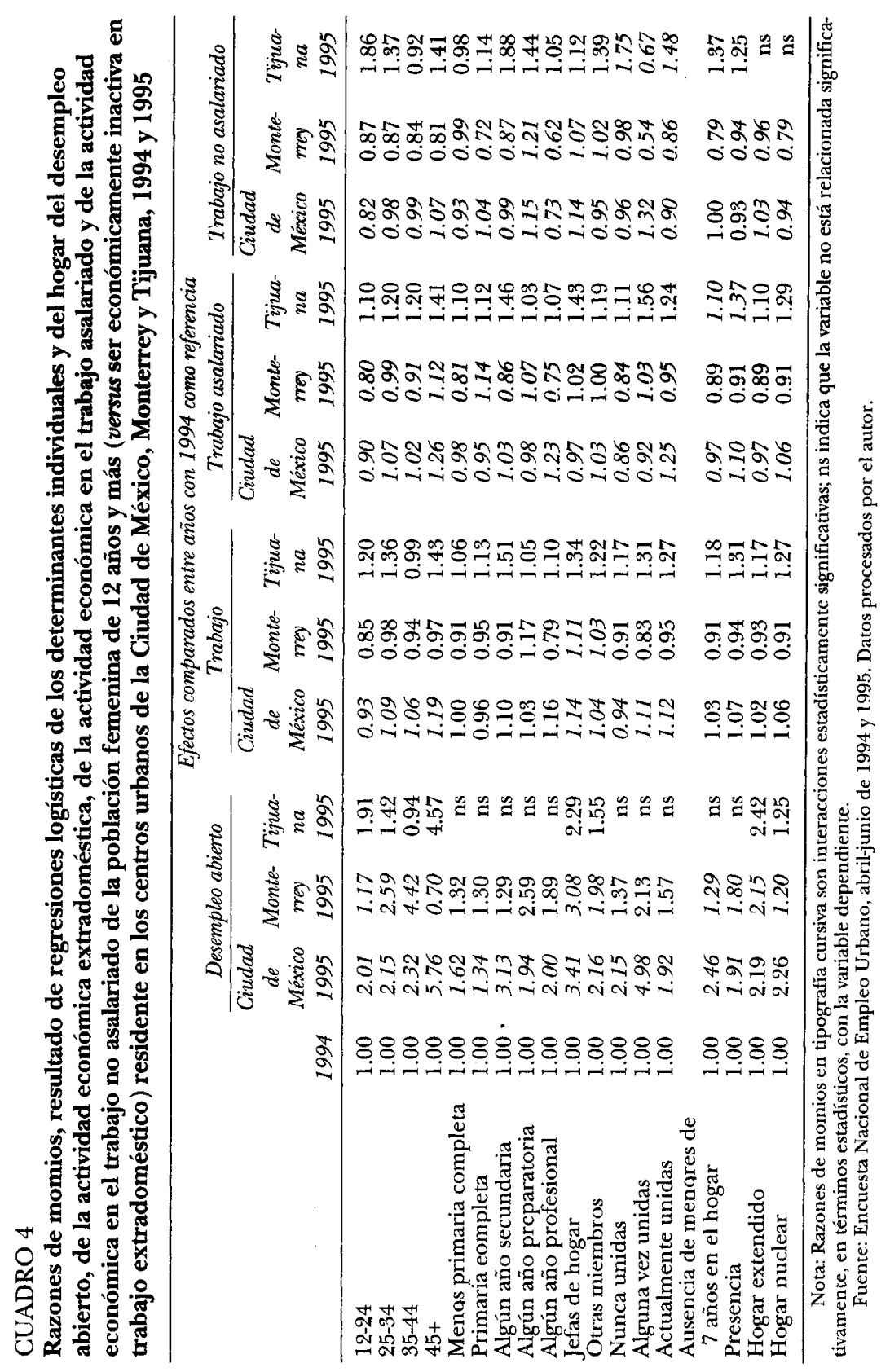


años de edad, fueron igualmente experimentados en contextos urbanos donde la participación femenina se mantuvo estable, aumentó o disminuyó. No obstante estos cambios, existen diferencias entre estas tres ciudades que vale la pena destacar, particularmente en el caso del desempleo abierto.

El desempleo abierto aumentó de forma generalizada en los tres centros urbanos. Sin embargo, los factores asociados con las probabilidades de estar desempleado en Tijuana variaron de manera significativa en relación con las otras ciudades. La educación, el estado marital y la presencia o ausencia de menores de siete años no fueron condiciones individuales asociadas con el hecho de estar buscando empleo en esta ciudad fronteriza. Esto indica la existencia de una dinámica de mercado de trabajo muy distinta en Tijuana, muy probablemente como resultado de la existencia de una estructura de oportunidades no sólo muy diversa, sino también poco estratificadora en términos de antecedentes tan importantes de las mujeres como la educación.

Con la excepción de las mujeres de 45 años y más en Monterrey, y de las de 35 a 44 años en Tijuana, el desempleo abierto se incrementó para las mujeres de todos los grupos de edades. Como se puede apreciar, y una vez que se toman en cuenta los efectos de otros factores de la oferta de mano de obra, no fueron las mujeres más jóvenes las que experimentaron los mayores aumentos en su propensión a estar desempleadas, sino las de edades más avanzadas. La información permite apreciar que las mujeres mayores de 45 años de edad fueron aquellas que más aumentaron sus niveles de desempleo abierto como consecuencia de la crisis económica en la ZMCM y Tijuana. Éste no fue el caso en Monterrey, donde este lugar fue ocupado por las mujeres de 35 a 44 años. Es evidente que fueron las mujeres en edades más avanzadas las que habían aumentado en mayor medida la búsqueda activa de trabajo durante los primeros meses de 1995. En la ZMCM y Tijuana las mujeres mayores de 44 años presentaron incrementos en sus razones de momios superiores a $400 \%$, y algo similar sucedió con las mujeres de 35 a 44 años en Monterrey.

Los efectos de la variable educación sobre el desempleo abierto fueron muy distintos en las tres ciudades. Como ya se mencionó, la educación no fue un factor determinante en las posibilidades de estar desempleada en Tijuana. Esto no sucedió en la ZMCM y Monterrey. El crecimiento del desempleo muestra situaciones distintas en estas dos äreas metropolitanas. En ambas ciudades las mujeres con primaria 
completa estuvieron entre aquellas que experimentaron menor aumento en el desempleo. No obstante, mientras que las mujeres con algún año de educación secundaria fueron las que más elevaron su desempleo en la ZMCM, esta posición fue ocupada por las mujeres con algún año de preparatoria en Monterrey. En general, es posible observar un mayor crecimiento del desempleo entre las mujeres con mayor instrucción formal; lo cual es un resultado esperado en vista de que éstas buscan preferentemente un trabajo asalariado, por un lado, y de que cuentan con mayores recursos económicos para costear periodos más largos de búsqueda activa de empleo.

Tal y como se observó en el agregado urbano, el desempleo abierto se expandió más entre las jefas de hogar que entre el resto de mujeres. Igualmente, pero con la excepción de Tijuana, las mujeres alguna vez unidas incrementaron más sus niveles de desempleo que las solteras y las actualmente unidas. Es importante destacar que en la capital del país el desempleo abierto creció un poco más entre las solteras que entre las actualmente unidas.

En Tijuana, la presencia de menores de edad en el hogar no es un factor sociodemográfico asociado estadísticamente con las posibilidades de estar desempleada. Sin embargo, en la ZMCM, las mujeres que residen en hogares donde no viven niños(as) pequeños(as), incrementaron su desempleo en mayor medida que aquellas que viven en hogares donde se requiere del cuidado de éstos. La situación en Monterrey fue distinta pues la presión económica en este centro urbano tuvo un mayor impacto sobre el desempleo de las mujeres que residen en hogares con niños pequeños.

Los resultados muestran que el desempleo abierto femenino creció tanto en los hogares nucleares como en los extendidos. Mientras que las diferencias entre las mujeres que residian en estos tipos de hogares es mínima en la ZMCM, en Tijuana y Monterrey las mujeres que habitaban hogares extendidos mostraron mayor crecimiento en sus propensiones de estar desempleadas.

¿Cuál fue entonces el impacto de la recesión sobre los niveles de ocupación de las mujeres? Efectivamente, la crisis económica tuvo como resultado un aumento significativo de las propensiones de las mujeres adultas de estar ocupadas en actividades económicas extradomésticas. Entre 1994 y 1995, las posibilidades de una mujer de 45 años o más de estar trabajando se incrementaron en $19 \%$ en la $\mathrm{ZMCM}$ y en $43 \%$ en Tijuana. En la capital del país, este aumento estuvo relacionado más significativamente con el trabajo asalariado que con el 
no asalariado (26 y $7 \%$, respectivamente); mientras que en la ciudad fronteriza no hubo diferencia alguna (41\%). En Monterrey el empleo de las mujeres de todas las edades disminuyó o se mantuvo estable en el periodo analizado. Sin embargo, es importante notar que el único incremento en el empleo femenino en esta ciudad norteña tuvo lugar entre las mujeres de más edad, cuyo trabajo asalariado se elevó en $12 \%$ (teniendo como contraparte una menor participación en trabajo no asalariado). La única excepción al mayor crecimiento del empleo entre las mujeres adultas avanzadas es Tijuana, pues en este centro urbano la propensión a trabajar de las mujeres másijóvenes fue superior. Este cambio se explica en gran medida por la gran demanda de las empresas maquiladoras para emplear mujeresijóvenes.

Los resultados muestran que en este contexto de crisis económica, y una vez controlados otros factores, no fueron las mujeres con el nivel educativo más bajo las que más expandieron sus niveles de ocupación. Tijuana es el único mercado de trabajo donde las mujeres con menos de la primaria completa aumentaron su empleo; sin embargo, éste estuvo entre los más bajos. En la ZMCM las mujeres con algún año de estudios profesionales fueron las que más aumentaron su propensión a trabajar, seguidas por las mujeres con algún año de secundaria. En contraste con la capital del país, en Monterrey las mujeres con mayor nivel educativo fueron las más afectadas por la crisis económica en lo que a sus niveles de ocupación se refiere. En esta ciudad, a pesar de que hubo una disminución en las oportunidades de empleo, las mujeres con algún año de preparatoria pudieron aumentar su empleo en 17 por ciento.

En Tijuana el empleo creció para las mujeres de todos los niveles educativos, pero se incrementó con mayor magnitud en aquéllas con algún año de secundaria. Como se puede observar, el incremento en la participación de estas mujeres no sólo ocurrió en el trabajo asalariado, como era de esperarse dada la gran demanda de las empresas maquiladoras por estas mujeres (46\%), sino también sucedió en el trabajo no asalariado ( 88 por ciento).

Los resultados para Monterrey son interesantes pues confirman una gran incorporación al mercado de trabajo de las mujeres con algún año de preparatoria. La población femenina con este nivel de instrucción fue la que más incrementó su desempleo abierto y, en un contexto de franco deterioro de los niveles de empleo femenino, fue la única que pudo aumentar su tasa de ocupación (17\%). La información de la ENEU arraja que, a pesar de que estas mujeres tienen 
una educación formal elevada para el contexto mexicano, su mayor participación fue más significativa en las actividades no asalariadas que en las asalariadas. El resto de mujeres, pero en especial aquéllas con educación profesional, mostró un franco deterioro en sus niveles de empleo.

En los tres contextos urbanos de participación femenina, y una vez controlado el efecto del resto de variables, tanto lasijefas de hogares como el resto de mujeres del hogar incrementaron sus niveles de ocupación. Sin embargo, en todos los casos lasijefas de hogar experimentaron en mayor medida su propensión a trabajar fuera del hogar. Existen diferencias importantes en cuanto a la relación de estos efectos con el trabajo asalariado y no asalariado. En la ZMCM, lasijefas de hogar mantuvieron casi un nivel constante de empleo en el trabajo asalariado, pero aumentaron su empleo no asalariado. El resto de miembros del hogar en la capital del país experimentó un proceso contrario. En Monterrey es claro que los aumentos insignificantes en la participación femenina de ambos grupos se debieron a las oportunidades encontradas en el trabajo no asalariado. En Tijuana lasijefas de hogares fueron las que sacaron mayores ventajas del crecimiento de las oportunidades económicas en el empleo asalariado.

Semejante a lo ocurrido en el agregado nacional urbano, en $\mathrm{Ti}$ juana y en la ZMCM la propensión a trabajar se elevó más entre las mujeres actualmente y alguna vez unidas que entre las solteras. Más aún, en esta última área urbana la ocupación de las solteras disminuyó. En la ZMCM puede apreciarse un proceso distinto entre las mujeres actualmente unidas y aquellas que alguna vez estuvieron unidas, pues mientras que el aumento en el empleo de las primeras se dio únicamente en oportunidades económicas asalariadas, el aumento en las segundas tuvo lugar sólo en el trabajo no asalariado. Es dificil explicar con la información disponible por qué las mujeres casadas o unidas, manteniendo constante la edad, la educación y el resto de factores, pudieron encontrar mayor acceso al trabajo asalariado que las mujeres alguna vez unidas. Monterrey muestra una historia distinta pues todas las mujeres disminuyeron su participación; sin embargo, este proceso ocurrió en menor medida entre las actualmente unidas.

Los resultados del cuadro 4 muestran claramente que en la ZMCM y en Tijuana, la presencia de niños pequeños en el hogar no fue un factor que impidiera a las mujeres incorporarse al mercado de trabajo durante este periodo de crisis económica. En la ZMCM, las mujeres que residían en hogares con menores de siete años de edad aumenta- 
ron su propensión a trabajar en $7 \%$ entre 1994 y 1995 , mientras que en Tijuana esta cifra se elevó hasta $31 \%$. Las magnitudes de estos cambios están indudablemente relacionados con aquellos observados en los niveles de empleo femenino en ambas ciudades. Una vez más llama fuertemente la atención el hecho de que el incremento en el empleo de estas mujeres tuvo lugar en mayor medida en el trabajo asalariado, pues incluso en la razón de momios descendió en el trabajo no asalariado en la capital del país. Monterrey mostró, al igual que en su patrón general de cambio, un descenso en la participación de mujeres que residen con o sin menores de edad.

Los resultados confirman la capacidad que tuvieron los hogares nucleares y extendidos para incrementar su oferta de trabajo femenino. Las historias son sin embargo distintas en cada ciudad. En la ZMCM, las mujeres residentes en hogares nucleares mostraron un mayor crecimiento de su propensión a trabajar que aquéllas que residían en hogares extendidos. Otra diferencia importante es que la inserción femenina de los primeros hogares tuvo lugar en el trabajo asalariado, mientras que la correspondiente a los segundos únicamente ocurrió en el trabajo no asalariado. En Tijuana las mujeres de ambos tipos de hogares aumentaron significativamente su participación, aunque esto sucedió en mayor medida en los nucleares. Al igual que en la ZMCM, el crecimiento en el empleo asalariado fue superior entre las mujeres de hogares nucleares que entre las residentes en hogares extendidos. Sin embargo, el tipo de hogar no estuvo significativamente relacionado con las posibilidades de empleo no asalariado en la ciudad fronteriza.

El mayor aumento en las propensiones de trabajar de las mujeres en hogares nucleares que en los extendidos manifiesta una aparente contradicción, pues estas últimas cuentan, en teoría, con un mayor apoyo en el interior de sus hogares para poderse incorporar a las mayoresijornadas de trabajo que demanda el empleo asalariado. Sin embargo varios factores pueden explicar este proceso. Primero, que los niveles de ocupación de los hombres en los hogares nucleares fueron mayormente afectados, y que por lo tanto las mujeres se vieron más obligadas a encontrar empleo. Segundo, que realmente los hogares nucleares no cuentan con menores apoyos sociales que los hogares extendidos en situaciones de crisis extrema. Tercero, que los hogares nucleares cuentan con recursos económicos superiores para consumir productos y servicios en el mercado en el caso de no tener acceso a redes sociales muy amplias. 


\section{Conclusiones}

A mediados de los años ochenta comenzaron a detectarse cambios muy importantes en los patrones de participación económica extradoméstica de las mujeres. Entre ellos destaca la creciente incorporación de mujeres que tradicionalmente habían mostrado muy baja propensión a formar parte de la fuerza de trabajo. Esta tendencia fue rápidamente identificada como resultado de la apremiante situación económica que muchas familias mexicanas vivieron durante el periodo de ajuste y restructuración económica. La persistencia de estos patrones de cambio en los años de recuperación macroeconómica, a finales de la década anterior y principios de la actual, fue crucial para sostener que el éxito de la política económica no era compartido por muchas familias mexicanas.

El propósito de este trabajo fue evaluar empíricamente el argumento que vincula un problema de bienestar familiar con el crecimiento del empleo de mujeres que históricamente habían mostrado probabilidades muy bajas de laborar fuera del hogar: adultas en edad avanzada, de bajo nivel educativo, casadas o unidas y al cuidado de niños(as) pequeños(as). A nuestroljucio, los cambios tan dramáticos que la economía nacional experimentó durante los primeros meses de 1995 ofrecían una excelente situación de coyuntura para validar esta proposición.

El análisis estadístico multivariado de los determinantes de la oferta de mano de obra femenina ha permitido comprobar que, con la única excepción de las mujeres con bajo nivel de instrucción formal, efectivamente estas mujeres "atípicas" mostraron una impresionante incorporación a la fuerza de trabajo en una situación de crisis económica aguda. Además, fue posible documentar que este incremento no fue sólo producto de una mayor propensión de estar trabajjando, sino también de probabilidades más altas de buscar activamente un empleo. El examen del tipo de inserción laboral permitió precisar, para nuestra propia sorpresa, que gran parte de la expansión del empleo de estas mujeres tuvo lugar en actividades económicas asalariadas. Este resultado pone de manifiesto que la demanda de trabajo pudiera estar jugando un papel significativo en la creciente salida al mercado de trabajo de mujeres adultas en edades avanzadas, unidas y con niños pequeños. Por lo tanto resulta necesario analizar más detalladamente el tipo de ocupaciones que estas mujeres están desempeñando y, a partir de esto, discutir sobre posibles factores que 
desde el lado de la demanda de empleo pudieran también explicar esta nueva dinámica laboral. Finalmente, el análisis de las áreas urbanas de la Ciudad de México, Monterrey y Tijuana ha permitido concluir que los patrones de incorporación descritos anteriormente ocurren en contextos de cambio muy variados en lo que respecta a los niveles de participación femenina.

\section{Bibliografía}

Beneria, Lourdes y Martha Roldán (1987), The Crossroads of Class and Gender: Industrial Homework, Subcontracting, and Household Dynamics in Mexico City, Chicago, The University of Chicago Press.

Cerrutti, Marcela (1997), "Coping with Opposing Pressure: A Comparative Analysis of Women Intermittence Participation in the Labor Force in Buenos Aires and Mexico City", tesis doctoral en sociología y demografia, Austin, Universidad de Texas.

Cruz Piñeiro, Rodolfo (1994), "Volatilidad en el empleo femenino: características individuales y del hogar", Frontera Norte, vol. 6, núm. 12, pp. 25-39.

Christenson, Bruce, Brígida García y Orlandina de Oliveira (1989), "Los múltiples condicionantes del trabajo femenino en México", Estudios Sociológicos, vol. 7, núm. 20, pp. 251-280.

Escobar, Agustín y Bryan R. Roberts (1991), "Urban Stratification, the Middle Classes, and Economic Change in Mexico", en M. González de la Rocha y A. Escobar (eds.), Social Responses to Mexico's Economic Crisis of the 1980s, San Diego, Center for U.S.-Mexican Studies, UCSD, pp. 91-113.

Fussell, Elizabeth y René Zenteno (1996), "Wage and Non-Wage Female Labor Force Participation in México: Is Export-Led Development Making a Difference in the Mexican Northern Border", trabajo presentado en la Reunión Anual de la Asociación de Estudios de Población de Estados Unidos (PAA), Nueva Orleáns, mayo (mimeo.).

García, Brígida (1989), "La importancia del trabajo no asalariado en la economía urbana", Estudios Demográficos y Urbanos, vol. 4, núm. 3 (12), pp. 439-463.

— y Orlandina de Oliveira (1992), "El trabajo femenino en México a fines de los ochenta”, en H. Muñoz (comp.), Población y sociedad en México, México, Coordinación de Humanidades, UNAM/Porrúa, pp. 243-267.

y Orlandina de Oliveira (1994), Trabajo femenino y vida familiar en Més xico, México, El Colegio de México.

- y Orlandina de Oliveira (1996), "¿Qué sabemos de nuevo sobre la participación femenina en los mercados de trabajo", México, El Colegio de México (mimeo.).

González de la Rocha, Mercedes (1994), The Resources of Poverty: Women and Survival in a Mexican City, Cambridge, Blackwell Publishers. 
Hualde, Alfredo y René Zenteno (1996), "Tijuana: ¿un mercado de trabajo en transición?", trabajo presentado en el I Congreso Mexicano de Sociología del Trabajo, Guadalajara (mimeo.).

Lustig, Nora (1990), "Economic Crisis, Adjustment, and Living Standards in Mexico, 1982-85", World Development, vol. 18, núm. 10, pp. 1325-1342.

Negrete, María Eugenia (1989), "Cambios en la estructura y distribución de la fuerza de trabajo en México: la dimensión regional, 1950-1980", en Memorias de la IIL.Reunión Nacional sobre la Investigación Demográfica en México, tomo 1, México, UNAM/Somede, pp. 621-641.

Oliveira, Orlandina de (1989), "La participación femenina en los mercados de trabajo urbanos en México: 1970-1980", Estudios Demográficos y Urbanos, vol. 4, núm. 3 (12), pp. 465-493.

___ y Brígida García (1997), “Crisis, restructuración económica y transformación de los mercados de trabajo en México", México, El Colegio de México (mimeo.).

—_ y Bryan Roberts (1993), "La informalidad urbana en años de expansión, crisis y restructuración económica”, Estudios Sociológicos, vol. 11, núm. 31, pp. 33-58.

Pacheco, Edith y Susan Parker (1997), "Male and Female Labor Market Mobility in México: Longitudinal Evidence From Two Periods of Crisis", trabajo presentado en la Reunión Anual de la Asociación de Estudios de Población de Estados Unidos (PAA), Washington, marzo (mimeo.).

Pozos, Fernando (1995), Restructuración y crisis económica en Guadalajara y Monterrey, Guadalajara, Universidad de Guadalajara.

Rubin-Kurtzman, Jane (1993), "¿Lecciones para el futuro? Cambios en los determinantes del empleo femenino en épocas de recesión en la Ciudad de México, 1970-1976”, Estudios Demográficos y Urbanos, vol. 8, núm. 3 (24), pp. 493-523.

Zenteno, René (1995a), "Del rancho de la tía Juana a Tijuana: una breve historia de desarrollo y población en la frontera norte”, Estudios Demográficos y Urbanos, vol. 10, núm. 1 (28), pp. 105-132.

(1995b), "On Depicting Local Labor Markets in Mexico: The Structure of Employment Conditions in Tijuana and Ciudad Juárez", tesis doctoral en sociología y demografia, Austin, Universidad de Texas. 